\title{
A Buffer Management Strategy based on Message Drop History in DTN Satellite Network
}

\author{
Li Yang ${ }^{a}$, Shuangjian Chen ${ }^{b}$ and Debin Wei ${ }^{c}$ \\ Communication and Network Lab, Dalian University, Dalian 116622, China. \\ ayangli945@126.com, b15382189177@163.com, cweidebin@163.com
}

Keywords: Buffer Management, Message Drop History, DTN

\begin{abstract}
In order to improve the performance of the DTN network, the DTN network routing algorithm improves the message delivery proportion and reduces the end-to-end delay by increasing the copy of messages. However, a large number of copies of the messages exist in the network, making the congestion of nodes with limited buffer. Therefore, an effective buffer management strategy is essential to improve the efficiency of DTN routing algorithm. In this paper, a buffer management strategy based on the history of message dropping is proposed. By counting the drop time and the number of drop times for each message arriving at their destination node in the message history list, to decide the order of dropping the message. Then, using the OPNET Modeler 14.5 to model the satellite nodes and building LEO satellite network simulation platform for simulation verification. The results show that, compared with other buffer management strategies, the proposed buffer management strategy based on message drop history can significantly improve the delivery proportion and reduce network overhead.
\end{abstract}

\section{Introduction}

Satellite networks have features like limited transmission energy, rapid mobility, frequent switching, sparse node density, frequent equipment failures, time-varying topology and so on [1]. So, satellite network is intermittent contact, which makes it form a typical delay/disruption tolerant network (DTN) [2, 3, and 4]. The DTN system adopts the message exchange mechanism of storagecarrying-forwarding [5] by adding a binding layer between the transport layer and the application layer. It is very important to carry out congestion control in resource-constrained satellite DTN networks. However, the research on satellite DTN is mainly focused on routing algorithm $[6,7$, and 8]. There is not much research congestion control algorithm in DTN satellite network.

At present, the main buffer management strategies at home and abroad as following: [9] tests and analyze the performance of dropping the longest queued message(Drop Head), dropping the newly received message(Drop Tail) and priori dropping the forwarded message by using Epidemic routing algorithm, points out that Drop Head has better performance than Drop Tail. It will increase delivery proportion but also increase the transmission delay to giving high priority for message in source node; in [10], A. Krifa et al. propose a scheduling drop strategy, which reduces the average transmission delay and increases the delivery proportion of messages in the network by calculating the probability of the message arrives at the destination node. But this strategy relies more on network topology information. At the same time, they also pointed out that dropping the messages with more number will reduce the delivery proportion but it is not actually. Because dropping the message is from the local considerations and from the global point of view, it will not have great impact on the delivery proportion; in [11], L. Yun et al. propose a new congestion control strategy, this algorithm determines whether to drop messages according to the forwarding times of messages in network. It significantly reduces the network overhead but under the condition of limited communication range, the performance of this algorithm is limited due to the different size of messages. In [12], Lindgren et al. study and analyze the PROPHET routing algorithm with a variety of buffer replacement strategies and buffer scheduling methods. The results show that a reasonable buffer management strategy can effectively improve the performance of routing algorithms. In [8], Erramilli et al. propose a strategy for dispatching, dropping and forwarding the messages based on DF (Delegation Forwarding) routing 
algorithm, in which messages get the different priority according to the distance of itself from the destination node. At the same time, Erramilli points out that the dropping or replacement of messages has more influence on the performance of routing algorithm than the dispatch of messages.

In conclusion, a buffer management strategy based on message drop history (DTM) is proposed considering the advantages and disadvantages of [10] and [11]. The simulation results show that DTM can obviously improve the efficiency of the buffer space, retain more useful messages, avoid invalid dropping, and take into account the fairness of message drop.

\section{Design of DTM Algorithm}

\subsection{Design Ideas of DTM Algorithm}

In order to effectively alleviate the congestion of the DTN node and reserve the forwarding message with the routing protocol, it's necessary to make the local node have memory ability in buffer management. The ability to remember here refers to the statistics of the message information in the local node, including the destination node of the message, the drop time, the number of drop times, and so on. The buffer management strategy based on the drop history is calculating the average time of historical drop by the formula according to the above statistics and storing the result in a list of messages. At last, the message in the buffer is queued according to the average time of historical drop in the message list. The longer the average time, the message is dropped earlier. In this case, the dropped message creates a memory in the node, which is associated with the destination node of the message. And whenever the node is congested, it is possible to drop the messages that are difficult to forward to the corresponding destination node in the local node.

In the initial state, the message in the local node $A$ that needs to be delivered to node $i$ is $j$, and the time at which the local node arrives is the time ${ }_{T}$. The time to drop the message due to the end of the life of the message or long queuing delay is ${ }^{T_{i j}}$, and the number of drop times is $\mathrm{N}$. Then the drop time interval for each messages is $\Delta_{i j}=T_{i j}-T_{i j}$. The historical average drop time for the messages to the destination node $\mathrm{i}$ is set as $\bar{\Delta}_{i}$, calculated as follows:

$$
\begin{aligned}
& \bar{\Delta}_{i}=\frac{\sum_{j=1}^{n} \Delta_{i j}}{\mathbf{N}} \\
& \bar{\Delta}_{i}=\frac{\bar{\Delta}_{i j}+\Delta_{i j+1}}{2}
\end{aligned}
$$

In the formula (1), $\mathrm{n}$ is the number of messages whose destination node is $\mathrm{i}$ in the local node $\mathrm{A}$ and $\Delta_{i+1}$ is the time interval of the next drop message. The calculated $\bar{\Delta}_{i}$ marks each messages in the buffer space and these messages queue according to $\bar{\Delta}_{i}$. Whenever it needs to drop the messages in buffer space, the priority drop the message with large value of $\bar{\Delta}_{i}$. Local node A makes full use of the historical information of the message in buffer space, searches for messages delivered to the same destination node, and then estimates the historical average time that these messages take in the buffer space. Dropping of the messages with long historical average time firstly greatly improve the efficiency of the use of buffer space, shorten the round trip delay of messages. The following figure shows the flow chart of DTM strategy.

\subsection{Feasibility Analysis of DTM Algorithm}

The topology of the DTN network is constantly updated. At the beginning of delivering the messages, the local node needs a certain time to accumulate the drop history of the messages. Therefore, the DTM sets the threshold of the number of messages dropped, that is, when the number of drop times of the message arrives at the same destination node $\mathrm{B}, \mathrm{N}$, is smaller than the threshold value, using the classic Drop Head buffer management policy; until $\mathrm{N}$ is greater than the threshold value, start the DTM buffer management policy.

As seen in section 2.1, as DTM runs, messages with the large value of $\bar{\Delta}_{i}$ are dropped. The dropping of the messages due to DTM results in the decreasing of ${ }^{\Delta_{i j}}$. As seen from formula (2), $\bar{\Delta}_{i}$ is also decreasing with it, and then the original priority to drop the message whose destination node is $\mathrm{j}$ 
are gradually retained. Therefore, this cyclical dynamic buffer management strategy not only takes advantage of the classic Drop Head buffer management strategy, but also increases the memory capability of the average time of the historical messages in local node. So that, without affecting the routing protocol, it effectively alleviate the node congestion due to limited buffer of local node, while takes into account the fairness of message drop, makes queuing of longer messages in the buffer of local node can also be delivered to the next node, rather than blindly drop.

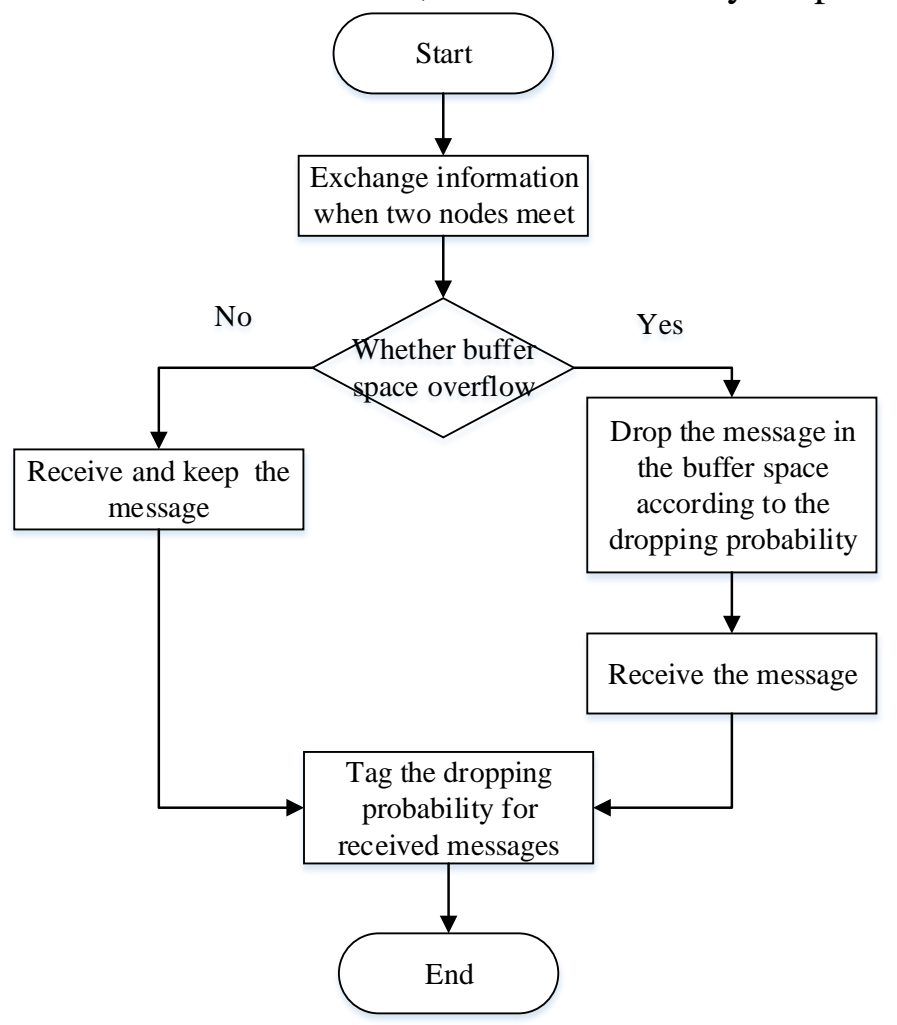

Fig. 1 Flow Chart of DTM Strategy

\section{Simulation and Analysis of Results}

The satellite DTN network studied in this paper is a communication environment where the internode links are interrupted easily and the end-to-end path is scarce. Therefore, when constructing loworbit satellite constellations, we design a smaller number of satellites in this paper. There are 9 satellites and 3 ground stations in the low-orbit satellite constellation simulation platform. We inspect the performance of the Drop Head algorithm, Drop Tail algorithm and DTM algorithm from the data delivery portion, the average end-to-end delay and the network overhead.

\subsection{Introduction of Simulation Environment}

Simulation tools include OPNET Modeler, STK and MATLAB. STK is responsible for the construction of LEO constellation, outputting the orbit parameters and location information of satellites and outputting the report of satellite intermittent; MATLAB is used to deal with prepredicted constellation data and formation of 0,1 topology matrix; OPNET Modeler is responsible for the overall network simulation, building core functional module of the DTN network.

\subsection{Design of Simulation in OPNET}

1) Design of Network Model

Building the satellite constellation structures with STK, specific parameters of constellation are shown is Table 1. Among them, nine LEO satellites follows walker distribution and three ground stations are located in Kashi, Bejing and Sanya. In order to reflect the purpose, the satellites are the source nodes to send data and the ground stations are the destination node to receive data. In this paper, the size of generated messages obeys Poisson distribution with the expected value of $128 \mathrm{~KB}$, and the interval of the messages is set to 0.5 seconds. 
Table 1 Scenario parameters

\begin{tabular}{cc}
\hline Parameters & Values \\
\hline Altitude of satellite orbit & $1680 \mathrm{~km}$ \\
Number of orbital plane & 3 \\
Number of satellites in each orbit & 3 \\
Inclination of satellite orbit & 60 \\
Link rate & $5 \mathrm{Mbps}$ \\
Ground station & Kashi, Beijing, Sanya \\
\hline
\end{tabular}

Building the network model as shown in Fig. 2 corresponds to satellite DTN network established in STK. There are 9 satellites using the same satellite node model. The "Config" node is responsible for defining the source and destination nodes of the messages.

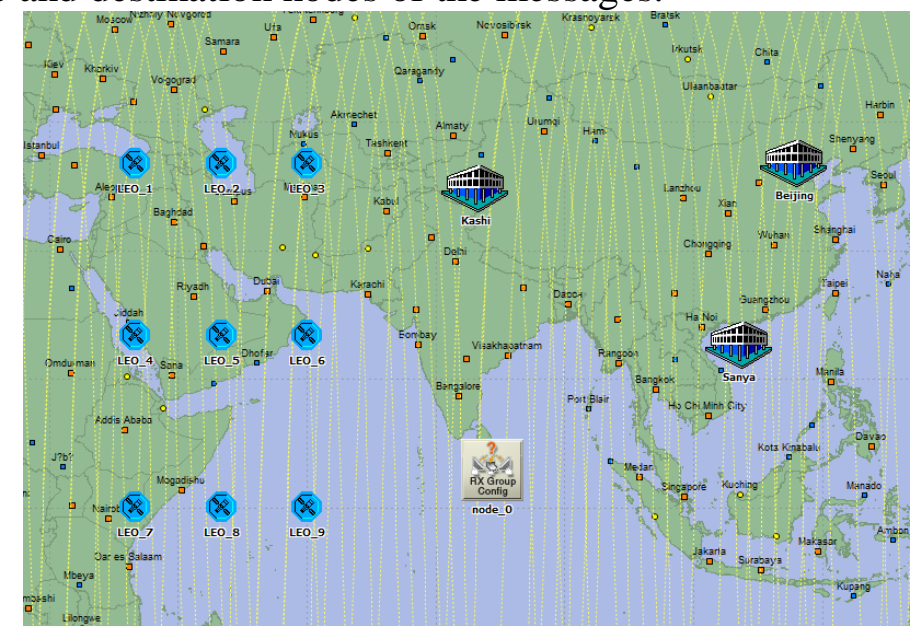

Fig. 2 Network Model

\section{2) Design of Node Model}

The source node of the message is set as the satellite node and the destination node is set as the ground station node. The satellite node and ground station node are respectively shown in Fig. 3(a), 3(b).

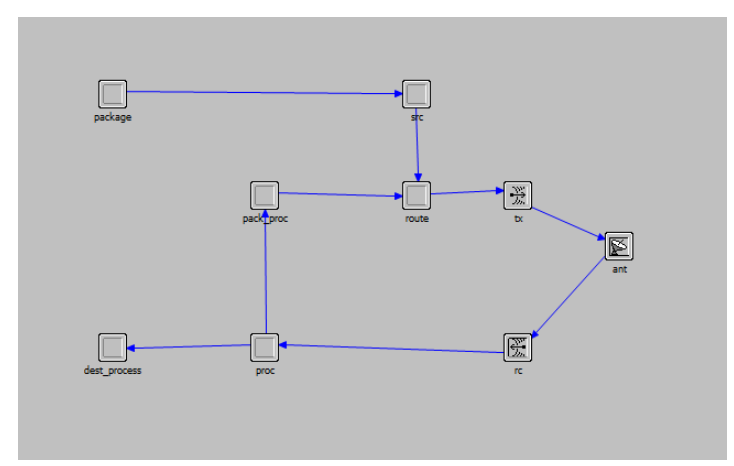

(a)

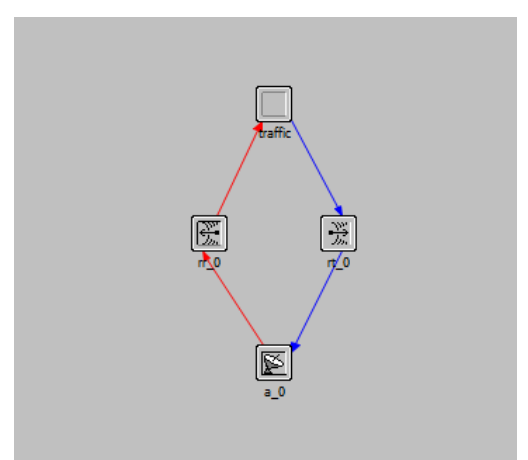

(b)

Fig. 3 Node Model

The satellite node model contains the processor "package" for generating the message, the processor "dest_process" for receiving messages, the processor "pack_proc" for managing the pack messages, the processor "route" for routing messages and so on. The ground station node contains only one receiving message processor "traffic", a pair of transceivers and an antenna model.

3) Design of Process Model

The core process model used in this simulation is shown in Figure 4. Figure 4(a) is the processor "traffic" of the ground station node, and figure 4(b) is the processor "pack_proc" of the satellite node. They are used for message reception on the ground station node and processing of messages queue buffer on satellite node. 


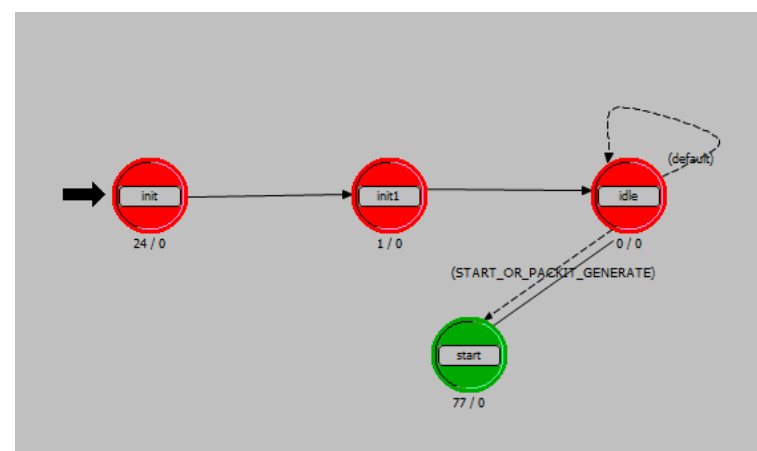

(a)

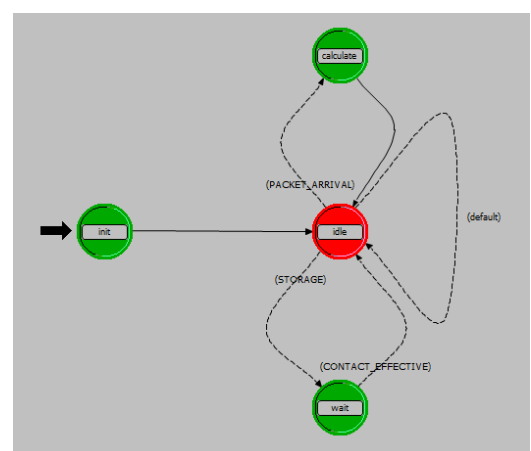

(b)

Fig. 4 Process Model

\subsection{Analysis of Simulation Results}

In the same network scenarios, we respectively carry out simulations for DTM algorithm, Drop Head algorithm and Drop Tail algorithm. By changing the cache size of nodes, we inspect the performance of each algorithm from the message delivery rate, average transmission delay and network overhead ratio. The message delivery rate $=$ number of delivery messages $/$ number of messages generated on source node; network overhead ratio $=$ (number of messages relayed in the network - number of delivery messages) / number of delivery messages, smaller network overhead means better performance of the network.

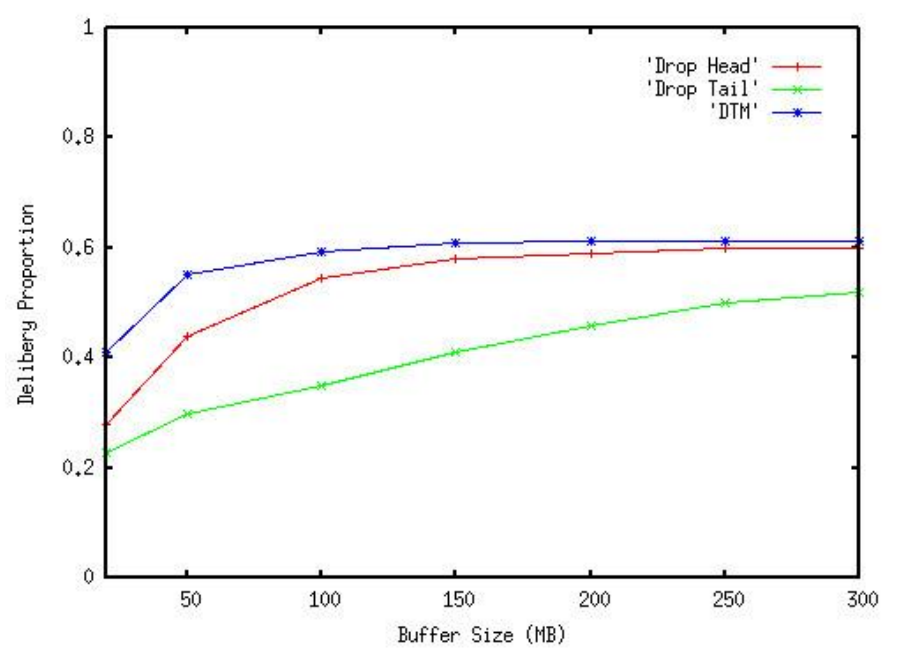

Fig. 5 Message delivery proportion and

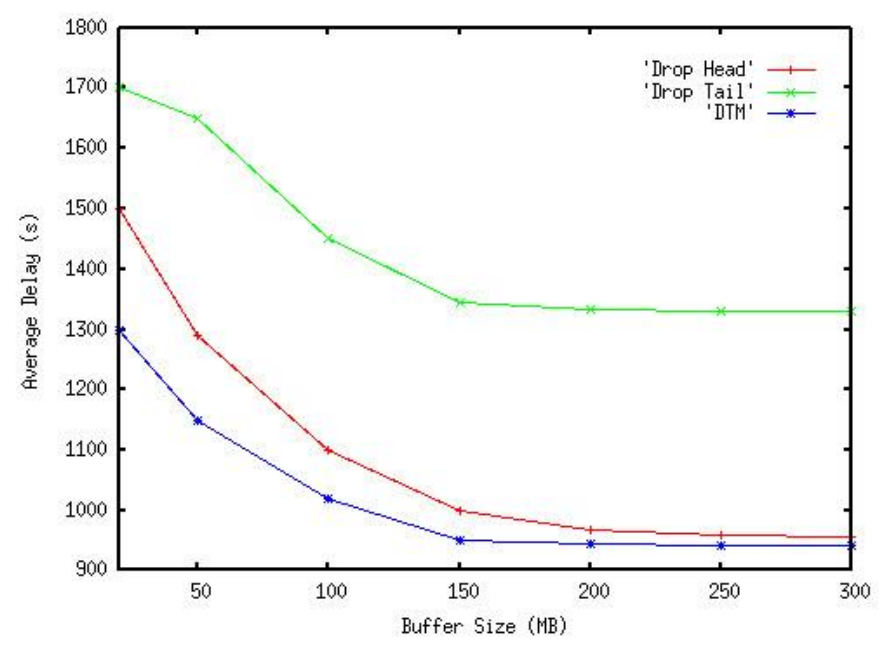

Fig. 6 Average transmission delay

As shown in Fig. 5, the message delivery proportion of Drop Head is higher than Drop Tail. When the buffer space of the node is less than $100 \mathrm{MB}$, the message delivery proportion of the DTM policy 
is significantly higher than that of the Drop Head and Drop Tail policies, which means that the DTM strategy can drop the message occupied the buffer for long time in the case where the node cache space is limited in time. And when the node's buffer space is greater than 100MB, the advantage of DTM strategy is not obvious.

As shown in Fig. 6, the average transmission delay of DTM strategy is better than the other two strategies and the performance of Drop Tail strategy is the worst. But the average transmission delay of the DTM strategy is close to Drop Head after the buffer capacity is greater than $150 \mathrm{MB}$. Therefore, the DTM strategy can drop the message occupied the buffer for long time in time when node congestion occurs, and reserve the messages with faster delivery, earlier to reach the destination node in the buffer space. It not only improves the message delivery proportion, but also shortens the average delivery delay of the messages.

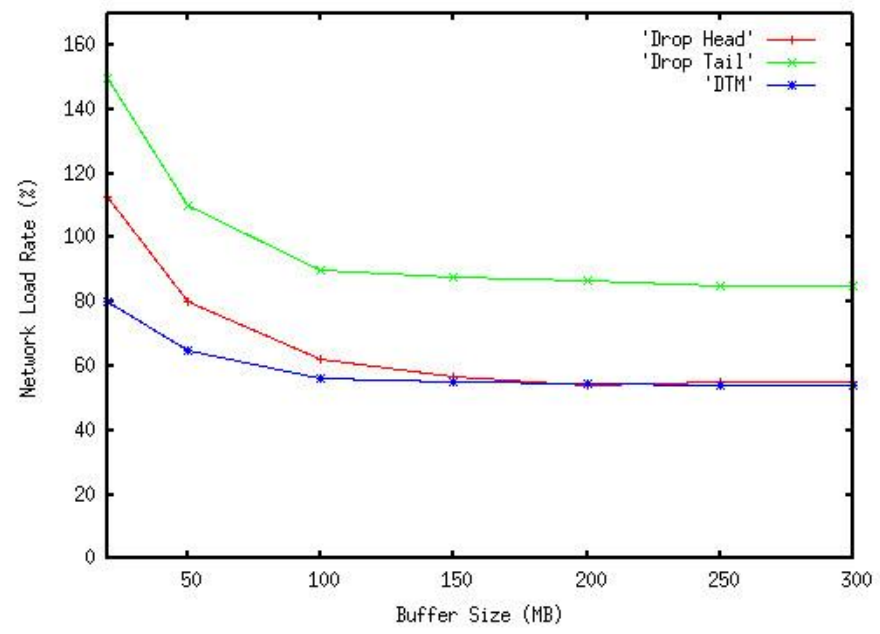

Fig. 7 Network overhead ratio

As shown in Fig. 7, when the buffer space of the node is less than 100MB, the network overhead ratio of the DTM strategy is much better than the other two kinds of strategies. When the buffer is larger than $100 \mathrm{MB}$, the buffer space of the node is abundant, and the number of messages that need to be dropped is less. Therefore, the proportion of the messages that have been delivered successfully increases, and the network overhead ratio of the three strategies tends to be stable. Because the DTM strategy retains more useful messages, avoiding invalid drop, the network overhead ratio is better than the other two strategies, about $23 \%$ lower.

\section{Summary}

In order to improve the performance of DTN network more effectively, a buffer management strategy based on message drop history is proposed in this paper, which drop the message with longer historical average time firstly, greatly improves the efficiency of buffer space, retains more useful messages, avoids invalid drop. The simulation results show that compared with the traditional buffer management strategy, this strategy can improve the performance of message delivery proportion, network average transmission delay and network overhead ratio. At the same time, the strategy can adjust the discard order according to the historical drop information of the network, and take into account the fairness of the message transmission and has a long adaptability.

\section{References}

[1]. Rao, Yuan, and Ru-chuan Wang. "Agent-based load balancing routing for LEO satellite networks." Computer networks 54.17 (2010):3187-3195.

[2]. Delay-tolerant networking architecture, RFC 4838[EB/OL]. http:// tools.ietf.org/html/rfc4838, 2015-12-01.

[3]. LIN C, DONG Y W, SHAN Z G. Research on space internetworking service based on DTN[J]. Journal of Computer Research and Development, 2014, 51(5): 931-943. 
[4]. ZHANG Z. Routing in intermittently connected mobile ad hoc net-works and delay tolerant networks: overview and challenges [J]. IEEE Communications Surveys \& Tutorials, 2006, 8(1): 24-37.

[5]. HOOKE A. The interplanetary Internet[J]. Communications of The ACM, 2001, 44(9): 38-40.

[6]. Bezirgiannidis N, Tsapeli F, Diamantopoulos S, et al. Towards flexibility and accuracy in space DTN Communications[C]. Proceedings of the 8th ACM MobiCom Workshop on Challenged Networks, Miami, Florida, USA, 2013: 43-48.

[7]. Fraire J and Finochietto J M. Routing-aware fair contact plan design for predictable delay tolerant networks [J]. Ad Hoc Networks, 2015, 25(Part B): 303-313.

[8]. Fraire J and Finochietto J M. Design challenges in contact plans for disruption-tolerant satellite networks [J]. IEEE Communications Magazine, 2015, 53(5): 163-169.

[9]. Zhang X, Neglia G, Kurose J, et al. Performance modeling of epidemic routing [J]. Computer Networks, 2007, 51(10): 2867-2891.

[10]. Krifa A, Barakat C, Spyropoulos T. Optimal buffer management policies for delay tolerant networks[C]. Proceedings of the 5th Annual IEEE Communications Society Conference on Sensor, Mesh and Ad Hoc Communications and Networks. Piscataway, NJ: IEEE, 2008. 260268.

[11]. Yun L, Xinjian C, Qilie L, et al. A novel congestion control strategy in delay tolerant networks[C]. Future Networks, 2010. ICFN'10. Second International Conference on. IEEE, 2010: 233-237.

[12]. Lindgren A, Phanse K S. Evaluation of queueing policies and forwarding strategies for routing in intermittently connected networks[C]. Communication System Software and Middleware, 2006. Comsware 2006. First International Conference on. IEEE, 2006: 1-10. 